\title{
Demographic drivers of generation time in a bird metapopulation: evolutionary potential and the ecological determinants of pace-of-life
}

\author{
Yimen Araya-Ajoy ${ }^{1}$, Alina Niskanen ${ }^{2}$, Peter Ranke $^{1}$, Hannah Froy ${ }^{1}$, Thomas Kvalnes ${ }^{1}$, \\ Bernt Rønning ${ }^{1}$, Michael Pedersen ${ }^{1}$, Thor-Harald Ringsby ${ }^{1}$, Henrik Jensen ${ }^{1}$, Bernt-Erik \\ Sæther ${ }^{3}$, and Jonathan Wright ${ }^{4}$ \\ ${ }^{1}$ Norwegian University of Science and Technology \\ ${ }^{2}$ University of Oulu \\ ${ }^{3}$ Centre for Biodiversity Dynamics \\ ${ }^{4} \mathrm{NTNU}$
}

June 17, 2020

\begin{abstract}
Generation time determines the pace of key demographic and evolutionary processes. Quantified as the weighted mean age at reproduction, it can be studied as a trait that may evolve and change in response to ecological conditions. We combined quantitative genetic analyses of individual projection matrices with age- and density-dependent models to study generation time variation in a bird metapopulation. We found that males have longer generation times than females and that it is a heritable trait. Individuals with longer generation times contributed to population growth later in life, lived longer, produced fewer recruits per year, had greater lifetime reproductive success, but not necessarily a higher expected individual growth rate. As predicted by density-dependence theory, generation times were shorter when populations were growing, and longer when populations were closer to equilibrium or declining. These results support classic theory predicting that competitive regimes are key determinants of the pace of life-history strategies.
\end{abstract}

Demographic drivers of generation time in a bird metapopulation: evolutionary potential and the ecological determinants of pace-of-life

Yimen G. Araya-Ajoy ${ }^{1 *}$, Alina K. Niskanen ${ }^{1,2}$, Hannah Froy ${ }^{1}$, Peter Sjolte Ranke ${ }^{1}$, Thomas Kvalnes ${ }^{1}$, Bernt Rønning ${ }^{1}$, Michael P. Pedersen ${ }^{1}$, Henrik Jensen ${ }^{1}$, Thor Harald Ringsby ${ }^{1}$, Bernt-Erik Sæther ${ }^{1}$, Jonathan Wright $^{1}$

${ }^{1}$ Centre for Biodiversity Dynamics (CBD), Department of Biology, Norwegian University of Science and Technology (NTNU), N-7491 Trondheim, Norway.

${ }^{2}$ Ecology and Genetics Research Unit, University of Oulu, 90014 Oulu, Finland

*Corresponding author: yimencr@gmail.com

Keywords: life-history theory, trade-offs, density dependence, age dependence, pace-of-life syndromes, fastslow axis.

Running title: Pace-of-life in a bird metapopulation

Type of article: letter

Abstract: 150/150 words; main text: 4966 words; references: 45, figures: 2, tables: 4 
Data accessibility statement: Data supporting the results will be archived in Dryad.

Statement of authorship: YAA wrote the first draft of the manuscript, and all authors contributed substantially to revisions. YAA and JW designed the study. The study population was established by BES, THR and HJ. HJ, THR, TK, PSR and BR conducted fieldwork. AN and HJ developed the pedigree. YAA perform the analyses with input from all co-authors.

Abstract (150/150 words)

Generation time determines the pace of key demographic and evolutionary processes. Quantified as the weighted mean age at reproduction, it can be studied as a trait that may evolve and change in response to ecological conditions. We combined quantitative genetic analyses of individual projection matrices with ageand density-dependent models to study generation time variation in a bird metapopulation. We found that generation time is a heritable trait and that males have longer generation times than females. Individuals with longer generation times contributed to population growth later in life, lived longer and produced fewer recruits per year. Moreover, these individuals had greater lifetime reproductive success, but not necessarily higher expected individual growth rates. Generation times were shorter when populations were growing, and longer when populations were closer to equilibrium or declining. These results support classic theory predicting that density dependence is a key determinant of the pace of life-history strategies.

\section{Introduction}

Generation time is a key parameter describing the pace of key biological processes. In age-structured populations, generation time is determined by age-specific survival and reproduction (Cochran \& Ellner 1992), so it has been suggested as a good descriptor of the pace of life-history strategies (Gaillard et al. 2005). Generation time is also in allometric relation with key characteristics of organisms such as its body size and its metabolic rate (Brown et al. 2004). It is related to the rate of mutation (Lehtonen \& Lanfear 2014) and the time a population needs to replace itself (Bienvenu \& Legendre 2015). Evolutionary responses to selection of a trait per unit time depend upon a population's generation time (Lande 1982), and as such it is a measure that connects the demographic and phenotypic characteristics of a population with the potential rate of evolutionary change. Furthermore, generation time is related to the susceptibility of organisms to stochastic fluctuations in the environment (Sæther et al. 2005) and it is a key component of evolutionary rescue models (Chevin et al. 2010). Understanding the ecological processes affecting the generation time is therefore essential for predicting the rate at which organisms can adapt to environmental change.

Generation time is generally defined as a population-level attribute. There are several definitions of generation time for age-structured populations, all of which can be calculated from survival and reproduction projection matrices (Cochran \& Ellner 1992; Bienvenu \& Legendre 2015). For instance, generation time has been defined as the time it takes a population to grow by a factor of its net reproductive rate (Coale 1972), and also as the average time between birth events in an ancestral lineage going from daughter to mother (Bienvenu \& Legendre 2015). Other definitions relate to the average age of mothers of newborns in the population (Charlesworth 1994), such as "mean age of the parents of offspring produced in a particular time period" (Cochran \& Ellner 1992), "the mean age at birth events for all individuals in a cohort" (Caswell 2001) or a generation (Steiner et al. 2014). Importantly, all formulations provide similar relative metrics when applied to a range of scenarios (Ellner 2018). In this study, we focus on understanding the evolutionary potential and ecological factors shaping generation time of individuals, measured as the weighted mean age of successful contribution to the breeding population (i.e. the mean age of recruit-producing parents weighted by the number of recruits they produce at each age; Cochran \& Ellner 1992; McGraw \& Caswell 1996).

Among-species comparisons have shown that generation time predicts the position of species on the fast-slow continuum of life-history covariation (Gaillard et al. 2005, 2016). At the fast end are organisms prioritizing current reproduction; that mature early, have high reproductive rates, short lifespans and short generation times. At the slow end of the continuum are organisms prioritizing survival over reproduction; characterized by high survival rates, low reproduction rates and long generation times. Generation time thus reflects how organisms resolve life-history trade-offs between current versus future reproduction, such as the trade- 
off between reproduction and survival (Gaillard et al.2005; Wright et al. 2019). Despite its evolutionary importance, surprisingly little research has focused on understanding the sources of within-species variation in generation time. This is probably because generation time has generally been considered as a static attribute at the population or species level. However, generation time as an individual-level life-history trait can be estimated from an individual's age dependent reproduction (McGraw \& Caswell 1996), and has been suggested as a potential measure of an individual's position on the fast-slow continuum of lifehistory covariation (Araya-Ajoyet al. 2018). Studies quantifying the sources of variation in generation time at the among-individual and among-population levels should provide important insights into its evolutionary potential and how it is shaped by ecological conditions (McGraw \& Caswell 1996; Araya-Ajoy et al. 2018; Wright et al. 2019).

Ecological conditions are expected to shape the generation time of organisms. The role of density dependence in determining the tempo of life-history strategies has been a long-standing research topic in evolutionary ecology (Pianka 1970; Stearns 1976; Boyce 1984; Wrightet al. 2019). Density dependence was introduced as a driver of life-history strategies in the context of $r$ - versus $K$-selection after island colonization (MacArthur $\&$ Wilson 1967). The general idea was that when populations are growing, density-independent selection will favor fast life-history strategies, but as populations approached their carrying capacity, density-dependent selection will favor slower life-history strategies. More recently, age-structured models of density-dependent evolution have been shown to provide general predictions concerning the factors that may affect the optimal age of reproduction (e.g. Engen \& Sæther 2016). Therefore, while generation time may be constrained by reproductive and developmental trade-offs, it can also be shaped by selection in response to ecological pressures.

Our aim in this study is to determine the evolutionary potential of generation time as a measure summarizing patterns of life-history covariation, and to assess the role of population dynamics in determining generation time. We studied variation in generation time in a metapopulation of house sparrows (Passer domesticus ) in northern Norway. We first estimated individual generation times based on individual projection matrices and decomposed its variation into genetic and environmental sources. Next, we studied the relationships between individual generation time and first age of successful reproduction, lifespan, reproduction rate, lifetime reproductive success and individual expected growth rates (McGraw \& Caswell 1996). We then studied how age- and density-dependent survival and reproduction in turn affected generation time. Finally, we studied how fluctuations in population growth affected the mean age at reproduction to test the prediction that when populations are expanding, generation times should be shorter, whereas when populations are decreasing or near their carrying capacity generation times should be longer.

\section{Methods}

Study system

This study was carried out in eight of the island populations in a metapopulation of house sparrows inhabiting eighteen islands in northern Norway $\left(66^{\circ} \mathrm{N} 13^{\circ} \mathrm{E}\right.$; see map in Baalsrud et al. 2014). The high recapture rates $(\sim 74 \%)$ in these eight study populations, combined with extensive sampling effort, allows close monitoring of the life-histories of most of the individuals inhabiting these islands. Each time a bird was handled, as nestling, fledged juvenile or adult, we took a blood sample for genetic analyses. A pedigree based on 605 highly polymorphic and independent single nucleotide polymorphisms (SNPs; Niskanen et al. 2020) was generated and then used for estimating the number of recruits produced per individual. We used specific data sets for the different analyses, but in general this metapopulation has been monitored continuously since 1993. However, systematic SNP genotyping of all adults started in 1998 in some islands and in 2003 for others. Therefore, the maximum possible time period of data included in these analyses differed between populations (Table S1).

\section{Estimates of individual life-history traits}

Based on the pedigree, we used the number of recruits produced by each individual in each year to calculate all the elements of individual projection matrices and derive some key life-history traits, such as individual 
expected growth rate and generation time (McGraw \& Caswell 1996). To calculate an individual's generation time, it must have produced a recruit. Thus, only individuals that produced at least one recruit during their lifetime were considered in these analyses of individual life-histories, resulting in a total of 1052 individuals (552 females and 500 males, see Table S1 for more details). From the individual projection matrix, we estimated individual generation time, measured as the weighted mean age at reproduction. As well as the expected individual growth rate, estimated as the dominant eigen value of the individual projection matrix (see Appendix for formulas). We also estimated the first age when an individual produced a recruit (first age), its lifespan, the average number of recruits per year (reproductive rate) and the total number of recruits produced during its life (lifetime reproductive success).

Modelling life-history (co)variation

We used univariate linear mixed-effect models to quantify the sources of variation in the individual estimates of generation time, age of first successful reproduction, lifespan, reproductive rate, lifetime reproductive success and individual growth rate (see Appendix S1B for model equations). All these models had sex as fixed effect and random intercepts for population identity $(\mathrm{n}=$ eight populations) and cohort (i.e. birth year; $\mathrm{n}=16$ cohorts). We assumed normally distributed errors in these analyses. As a second step, we fitted a multivariate model to study the phenotypic covariance among individuals in the different life-history traits (see Appendix S1C for equations). This model did not contain any random or fixed effects as we were interested in estimating the overall phenotypic covariance among individual life-history traits regardless of the cause. We thus estimated the 6x6 "residual" variance-covariance matrix.

We also fitted an animal model (Kruuk 2004) to decompose individual differences in generation time into additive genetic variation versus environmental variation (see Appendix S1C for more details). This model only had sex as fixed effect and cohort year as random effects and we assumed normal error distribution. The pedigree consisted of 3116 individuals with a mean of 4.6 ancestral generations.

Age and density dependent reproduction and survival

For the age- and density-dependent models, we utilized annual data on reproduction and survival for all individuals present within the studied time periods, regardless of whether they had produced a recruit or not (Table S1). In total, there were 5247 records of 2729 individuals (1325 females and 1361 males). We studied how annual recruit production and survival resulted in the observed means and (co)variance of individual life-histories by building age- and density-dependent reproduction and survival models.

We modelled annual survival and annual number of recruits of individuals using generalized linear mixedeffect models. Survival was modelled assuming a binomial error distribution and the annual number of recruits was modelled assuming an over dispersed Poisson error distribution. Both models had the same fixed and random effect structure, however the residual variance of the survival model was fixed to 1 . We included as fixed effects, sex and age as a two-level categorical variable $(0=$ first year breeders and $1=$ older individuals). We also fitted an interaction between sex and age, as we were expecting sex-specific patterns of age-dependent reproduction and survival (Stubberud et al. 2017). These models also had the mean population size and the annual deviations from the mean population size as fixed effects. This within-subject centering approach allowed us to model density regulation accounting for differences in the mean population size between populations, and allowed us to test for any spatial versus temporal effects of population size in recruitment and survival (van de Pol \& Wright 2009). We fitted year, population and individual as random effects. We then proceeded to extend these models and fitted a multivariate mixed-effects model, where we estimated the covariance between yearly survival and recruit production at the individual and residual level (see Appendix S1C for model equations).

Effects of population dynamics on the mean age of reproduction

To explore how population dynamics affected the mean age of parents of recruits each year in each population, we utilized annual data on reproduction and survival for all adult individuals within the studied time periods (Table S1). From this data we estimated the weighted mean age of the parents reproducing in a year for 
each population. This was estimate as the mean age of the successfully reproducing parents weighted by the number of recruits they produced separately (see appendix S1 for formulas). We estimated the weighted mean age at reproduction in a population each year for males and females. We then fitted a mixed-effect model that had as response variable the weighted mean age of reproducing individuals in a given year in a given population and as fixed effects sex and the mean and annual deviations of population size to distinguish between effects of spatial versus temporal fluctuations in population size on the mean age at reproduction of a population.

To further examine how the weighted mean age at reproduction was related to the ecological factors determining population growth, we fitted another mixed-effect model where the mean age at reproduction was also fitted as a response variable and the mean fitness of the population in each year and sex as fixed effects. We estimated the fitness of each individual in a given years as survival plus half the number of recruits contributed to the next year, because, in the absence of dispersal, this metric of fitness directly connects to local population dynamics of sexually reproducing species (Sæther \& Engen 2015). Importantly, this measure of fitness will determine the changes in population size across years that are not caused by immigration and emigration. Importantly, the mean fitness in the population in a given year directly connects to the expected population growth and should reflect current levels of competition in the population (Sæther \& Engen 2015), either because of variation in environmental conditions and/or due to variation in population density relative to the amount of resources. To control for the effects of age structure in determining the mean age at reproduction, we also fitted the two above mentioned models including the mean age of all the adults breeding in the population as an additional fixed effect.

\section{General modelling procedures}

We fitted linear mixed-effect models using a Bayesian framework implemented in R v3.3 (R Core Team 2018) with the package MCMCglmm (Hadfield 2010). We ran 1,100,000 iterations per model, from which we discarded the initial 100,000 (burn-in period). Each chain was sampled at an interval of 500 iterations, which resulted in low autocorrelation $(<0.05)$ among thinned samples. Posterior modes, $95 \%$ credible intervals $(\mathrm{CI})$ and (co)variances were estimated across the thinned samples for the fixed and random effects. Fixed-effect priors were normally distributed and diffuse with a mean of zero and a large variance (100). We explored the sensitivity of the variance-covariance matrix to the choice of prior. See Appendix S1 for prior details on each of the analyses. Mean values of the posterior distributions were robust to different relatively uninformative priors. However, the width and mode of the posterior distribution for the animal model was susceptible to prior choice. We thus decided to present the animal model results estimated with restricted maximum likelihood framework using the package ASreml-R v.4.

\section{Results}

\section{Sources of variation in individual life-histories}

Individual generation time in this metapopulation has a mean of $1.77(\mathrm{SD}=0.90)$ and ranged from 1 to 5.6 years. They were on average 0.20 years longer for males compared with females. Males also lived longer, started contributing to the adult population later in life than females and produced on average 0.10 fewer recruits per year (Table 1). Among-population differences and among birth year differences each explained around $5 \%$ of the variation in individual estimates of generation time (Table 1).

Quantitative genetic analyses estimated an additive genetic variance of $0.04(\mathrm{SE}=0.03)$ and a log-likelihood ratio test supported the hypothesis of non-zero additive genetic variance. Additive genetic variance explained around $4.8 \%$ ( $\mathrm{SE}=3.2$ ) of the variation in individual estimates of generation time, which translates into an evolvability (i.e. mean scaled additive genetic variance) of $1.27 \%$ ( $\mathrm{SE}=1.29)$. See Table $\mathrm{S} 2$ for the full results of the animal model.

\section{Covariations between life-history traits}

Individuals with longer generation times started reproducing later, lived longer and produced fewer recruits per breeding season compared to individuals with shorter generation times (Figure 1A-C, Table 2). In addi- 
tion, individuals with longer generation times had higher lifetime reproductive success, but not necessarily higher individual growth rates (Figure 1D and E, Table 2). This is partly explained by individual growth rate being a metric that puts more weight on reproduction earlier in life (McGraw \& Caswell 1996). Consequently, we found that individuals with the same lifespan could have quite different generation times, with different individuals reproducing much earlier or later relative to their lifespan (Figure 1B). There was a negative covariance between lifespan and reproductive rate, implying that individuals that lived longer were unlikely to produce many recruits per breeding attempt (Figure $1 \mathrm{~F}$ ). Individuals with greater lifetime reproductive success were those that lived longer, produced more recruits per breeding attempt, had longer generation times and had higher expected individual growth rates (Table 2).

\section{Survival and reproduction}

Both males and females produced fewer recruits in their first year of breeding (Table 3). Males produced, on average, fewer recruits in their first breeding year than females (first year fecundity rates: 0.27 for males and 0.33 for females). However, from their second year onwards, males produced more recruits than females per breeding attempt (fecundity rates: 0.57 for males and 0.47 for females). Males also had a higher survival probability than females on average ( 0.50 for females and 0.57 for males; Table 3$)$. Thus, in total males had longer generation times, both because they lived longer and because they were more successful at reproducing when they were older (Figure S2). In addition, we found evidence for strong density regulation in recruit production, but not in adult survival (Table 3). In years when population size was relatively high compared to the average population size, recruitment was lower (Table 3). However, there was a trend suggesting that in populations where population sizes were on average higher individuals produced more recruits (Table 3 ). We further explored whether these density-dependent patterns were sex and age specific, but we did not find any evidence for this.

We found a positive correlation between survival and reproduction among individuals (0.25; CI 0.15- 0.34) and within individuals across years (0.20; CI 0.08- 0.33). This suggests that consistent variation in phenotypic "quality" and/or resources among individuals resulted in some individuals producing, on average, more recruits per year and at the same time being on average more likely to survive. In addition, in years when individuals produced more recruits they were also more likely to survive. These results seem to contradict the observed negative covariance between lifespan and reproductive rate. We therefore used simulations to address this issue (see Appendix S3), finding that the negative covariance between reproduction rate and lifespan, and between reproduction rate and generation time, was the result of the age-dependent patterns of survival and reproduction, and not necessarily caused by individual resource allocation trade-offs (see Discussion).

\section{Mean age at reproduction and population growth}

The average age of fathers of all the recruits produced in this house sparrow metapopulation was 2.24 years $(\mathrm{SD}=1.29)$, while the average age of the mothers of all the recruits was $2.06(\mathrm{SD}=1.32)$. These metapopulation generation times are higher than the mean of the individual generation times (Table 1), because the meta-population generation time is heavily influenced by the individuals that reproduced the most. The higher estimates for the meta-population generation time imply that individuals that reproduced when they were, on average, older tended to contribute more recruits to the meta-population.

In years when the mean fitness of the population was lower, the mean age of successfully reproducing individuals was older (Table 4, Figure 2). This suggests that in years when competition was high and/or environmental conditions were bad, and so individuals had on average lower fitness, the successfully reproducing males where amongst the older males. In contrast, when the mean fitness of the population was high, and thus populations are expected to grow, the average age of reproducing males was younger. These effects cannot be solely attributed to differences in age structure, because even after correcting for the mean age of all the adults present, there was evidence that these effects were still different from zero (Table 4, model 1B). Supporting this findings, we also found a trend suggesting that in years when population size was higher than average, the mean age of reproducing males was older (Table 4). 


\section{Discussion}

Our results show that individual generation time measured as the mean age at reproduction summarizes the pace of an individual's life-history and has the potential to evolve in response to ecological pressures. Male generation time was longer compared to females due to sex differences in age-dependent reproduction and survival, likely caused by differences in competitive regimes. We also found that in years where competition was higher and average fitness was lower, recruit production was lower and older individuals contributed relatively more to population growth. In contrast, when populations were growing, younger individuals contributed disproportionately more to population growth. These results imply that fluctuations in competitive regimes associated to population sizes, influence the mean age at reproduction, thereby affecting the demographic characteristics of this metapopulation.

\section{Evolution of pace-of-life}

Generation time can be used to predict a species' position along the slow-fast continuum of life-history variation (Gaillard et al.2005; Araya-Ajoy et al. 2018). Classic life-history theory (Charlesworth 1994) suggests that variation in the mean age at reproduction evolves as consequence of compromises between age-specific survival and reproduction, resulting in some combination of life-history traits that optimizes mean fitness under particular ecological conditions. The contemporary evolution of the generation time, and thus the pace-of-life of an organism, hinges upon there being genetic variation underpinning the traits that determine generation time. When we estimated the additive genetic variation in individual measures of generation time, we found that evolutionary potential or evolvability was within the range of values estimated for other life-history traits (Hansen et al. 2011). Individuals with longer generation times had greater lifetime reproductive success, but not necessarily a greater individual growth rate, and thus it is not clear that selection should favor the evolution of longer or shorter generation times.

The evolution of generation time is predicted to be constrained by trade-offs between reproduction and survival (Stearns 1992; Roff 1993). In this study, we found the expected negative covariance between reproductive rate and generation time, and between reproductive rate and lifespan (Figures $1 \mathrm{~B}$ and $1 \mathrm{~F}$ ). This is partly unexpected, because variation in acquisition of resources is expected to mask life-history trade-offs in observational studies (van Noordwijk \& de Jong 1986). To further explore resource acquisition versus allocation in our metapopulation, we analyzed the patterns of covariation between individual lifehistory traits using principal component analyses (Table S3). We found that the most important driver of the first axis of variation was lifetime reproductive success, with positive loadings for reproductive rate and lifespan, reflecting among-individual variation in resource acquisition. Whereas the strongest driver of the second principal components axis was generation time, where the opposing signs of reproductive rate and life span revealed the classic survival versus reproduction trade-off.

To further explore evidence of allocation trade-offs, we partitioned the covariance between annual reproduction and survival at the among- and within-individual levels (Table 3). If there was a trade-off between survival and reproduction, then in years where individuals invested more in survival, they cannot have invested a lot in reproduction. This should generate a negative within-individual covariance due to resource allocation trade-offs. Variation among individuals in resource acquisition should, in turn, be manifested as a positive among-individual covariance, where individuals with higher than average recruit production will also have a greater chance of surviving, because they are able to acquire more resources. We found a positive covariance between survival and reproduction at both levels, and therefore we find no evidence of an allocation trade-off at the within-individual level.

The positive covariance between survival and annual reproduction partly appears to contradict the results above concerning the trade-off between lifespan and recruitment rate. We addressed this contradiction using statistical simulations based upon the estimates of annual survival and reproduction (see Appendix S3). We explored how the demographic characteristics of this metapopulation could have resulted in a negative covariance between lifespan and reproductive rate, in the presence of a positive covariance between survival and annual reproduction (see Figure S3). We found that even when there was variation among individuals 
in acquisition of resources, causing a positive covariance between survival and annual reproduction at the within- and among-individual levels, the resulting distribution of individual life-histories in the population tended to generate a negative covariance between lifespan and reproductive rate. Given the observed patterns of age- and density-dependent survival and reproduction, even when individuals that had a greater survival probability also had a higher reproduction rate, a negative correlation between lifespan and reproduction rate was possible, simply as a result of the distribution of individual life-histories in the population.

\section{Sexual dimorphism in mean age at reproduction}

We found that males contributed more to population growth when they were older compared to females (Table 1). Male house sparrows in our metapopulation lived longer and had longer generation times as compared to females (Table 1). Furthermore, we found that male production of recruits in their first year was lower compared to females, corroborating similar results for a larger sample of house sparrow populations (Stubberud et al. 2017). Due to less costly production of male gametes, male fitness is hypothesized to be largely limited by access to fertilizations. Males are thus predicted to invest more in reproduction through male-male competition at the expense of survival (Liker \& Szekely 2005; Bonduriansky et al. 2008). Our results do not support these predictions; we found that males lived longer and started reproducing later compared to females.

Sex differences in generation time can be explained by intra-sexual competition for nest sites or mates. For instance, lower production of recruits by males during their first breeding year could also be caused by more males being unpaired in their first breeding year because of a biased sex ratio. However, we did not find strong systematic bias in the sex ratio in this metapopulation. Another potential cause of sex differences in the mean age at reproduction could be associated to extra-pair reproduction (Hsu et al. 2017; Girndt et al.2018). If older males increased their reproductive output through extra-pair fertilizations, this would have contributed to the sex differences in the mean age at reproduction in our population. This is interpretation is partly supported by findings in this meta-population where traits that vary among male age classes (body mass, wing length and badge size; Araya-Ajoy et al. 2019) positively affect the number of mates (Jensen et al. 2008).

\section{Population dynamics and mean age at reproduction}

In our metapopulation of house sparrows, annual variation in population size and the strength of densitydependent intra-specific competition appears to generate temporal variation in the mean age at reproduction. If individuals produce the same number of recruits, and in the absence of density regulation, it is expected that individuals that reproduce earlier in life and thus have shorter generation times will be selected for, because early reproduction will result in a higher individual growth rate. Our results suggest that population dynamics affects the mean age at reproduction because density-dependent competition constrains the reproductive output of younger individuals. This is therefore consistent with the idea that density-dependent competition constrains individual growth rates. Especially for males, older individuals seem to contribute more to population growth when intra-specific competition is stronger.

We found strong support for density regulation in the production of recruits. In years when population sizes were higher than average, individuals produced fewer recruits. The patterns of density regulation in recruitment could have been mediated by effects on the reproduction of parents or via differential juvenile survival. Thus, in years where population sizes were higher than average, individuals fledged fewer offspring and/or juvenile survival was lower. In our study, we did not find that adult survival was density dependent, but given that our measure of first-year recruitment combines investment in reproduction by the parents with the survival probability of their offspring, then juvenile survival may well represent the point in the life-histories of these house sparrows where survival is most closely density regulated.

When we analyzed how the mean age at reproduction was affected by population dynamics across years and populations, we found a trend suggesting that in years where population sizes where larger than average then the mean age at reproduction was older (Table 3). We also analyzed how the expected growth of the population, measured as the mean fitness of the population in a given year, affected the mean age 
of the reproducing parents. We found that when the mean fitness of the population was low then the individuals that managed to reproduce were older (Table 4, model 1A). In contrast, when populations were growing either all individuals, even the young ones, managed to reproduce or individuals that invest more in current reproduction at the expense of future reproduction or survival were able to contribute disproportionately to population growth, as predicted by density-dependent selection theory (MacArthur \& Wilson 1967; Engenet al. 2013; Engen \& Saether 2016). Further analyses showed that this was not solely the result of the age structure of the population (Table 4, model 1B). These results are thus consistent with classic density-dependent selection theory predicting that in scenarios with stronger competition, the individuals being favored will be the ones that invest more in future reproduction (e.g. by investing in traits that increase survival and enable them to reproduce later). But is also consistent with the idea that 'high-quality' individuals are the ones that manage to grow old and can reproduce when density-dependent competition is strongest.

\section{Conclusions}

This study highlights the importance of integrating different approaches used to study life-history variation in order to gain a better understanding of the ecological causes and ultimately the evolutionary consequences of variation in the tempo of life-history strategies (i.e. pace-of-life). By combining an analysis of individual variation in life-histories based upon individual projection matrices, along with age- and density-dependent survival and reproduction models, we were able to understand the genetic and demographic sources of individual variation in generation time within a metapopulation of house sparrows. Furthermore, we show how competitive regimes can affect the mean age at reproduction, supporting predictions from classic lifehistory theory predicting that density dependence is a key determinant in shaping the pace-of-life. Because generation time determines the speed of evolutionary responses to selection, detailed understanding of how ecological dynamics affect the mean age at reproduction is therefore a key to predicting evolutionary adaptations to environmental change.

\section{References}

Araya-Ajoy, Y.G., Bolstad, G.H., Brommer, J., Careau, V., Dingemanse, N.J. \& Wright, J. (2018). Demographic measures of an individual's "pace of life": fecundity rate, lifespan, generation time, or a composite variable? Behav. Ecol. Sociobiol. , 72.

Araya-Ajoy, Y.G., Ranke, P.S., Kvalnes, T., Ronning, B., Holand, H., Myhre, A.M., et al. (2019). Characterizing morphological (co)variation using structural equation models: Body size, allometric relationships and evolvability in a house sparrow metapopulation.Evolution , 73, 452-466.

Baalsrud, H.T., Saether, B.E., Hagen, I.J., Myhre, A.M., Ringsby, T.H., Parn, H., et al. (2014). Effects of population characteristics and structure on estimates of effective population size in a house sparrow metapopulation. Mol. Ecol. , 23, 2653-2668.

Bienvenu, F. \& Legendre, S. (2015). A new approach to the generation time in matrix population models. Am. Nat. , 185, 834-843.

Bonduriansky, R., Maklakov, A., Zajitschek, F. \& Brooks, R. (2008). Sexual selection, sexual conflict and the evolution of ageing and life span. Funct. Ecol. , 22, 443-453.

Boyce, M.S. (1984). Restitution of r- and K-Selection as a Model of Density-Dependent Natural Selection. Annu. Rev. Ecol. Syst. , 15, 427-447.

Brown, J.H., Gillooly, J.F., Allen, A.P., Savage, V.M. \& West, G.B. (2004). Toward a metabolic theory of ecology. Ecology , 85, 1771-1789.

Caswell, H. (2001). Matrix Population Models: Construction, Analysis, and Interpretation . Matrix Population Models: Construction, Analysis, and Interpretation. Sinauer Associates. 
Charlesworth, B. (1994). Evolution in Age-Structured Populations . Cambridge Studies in Mathematical Biology. 2nd edn. Cambridge University Press.

Chevin, L.M., Lande, R. \& Mace, G.M. (2010). Adaptation, plasticity, and extinction in a changing environment: Towards a predictive theory.PLoS Biol. , 8.

Cleasby, I. \& Nakagawa, S. (2012). The influence of male age on within-pair and extra-pair paternity in passerines. Ibis (Lond. 1859). , 154, 318-324.

Coale, A.J. (1972). Growth and Structure of Human Populations: A Mathematical Investigation . Princeton University Press.

Cochran, M.E. \& Ellner, S. (1992). Simple Methods for Calculating Age-Based Life History Parameters for Stage-Structured Populations.Ecol. Monogr. , 62, 345-364.

Ellner, S.P. (2018). Generation time in structured populations.Am. Nat. , 192, 105-110.

Engen, S., Lande, R. \& Saether, B.-E. (2013). A Quantitative Genetic Model of r- and K-Selection in a Fluctuating Population. Am. Nat. , 181, 725-736.

Engen, S. \& Saether, B.E. (2016). Optimal age of maturity in fluctuating environments under r- and Kselection. Oikos, 125, 1577-1585.

Gaillard, J.-M., Lemaitre, J.-F., Berger, V., Bonenfant, C., Devillard, S., Douhard, M., et al. (2016). Life Histories, Axes of Variation in . Encycl. Evol. Biol.

Gaillard, J.-M., Yoccoz, N.G., Lebreton, J.-D., Bonenfant, C., Devillard, S., Loison, A., et al. (2005). Generation time: a reliable metric to measure life-history variation among mammalian populations. Am. Nat. , 166, 119-123; discussion 124-128.

Girndt, A., Chng, C.W.T., Burke, T. \& Schroeder, J. (2018). Male age is associated with extra-pair paternity, but not with extra-pair mating behaviour. Sci. Rep. , 8, 8378.

Hadfield, J.D. (2010). MCMC Methods for Multi-Response Generalized Linear Mixed Models: The MCMCglmm R Package. J. Stat. Softw. , 33, 1-36.

Hansen, T.F., Pelabon, C. \& Houle, D. (2011). Heritability is not Evolvability. Evol. Biol. , 38, 258-277.

Hsu, Y.-H., Simons, M.J.P., Schroeder, J., Girndt, A., Winney, I.S., Burke, T., et al. (2017). Age-dependent trajectories differ between within-pair and extra-pair paternity success. J. Evol. Biol. , 30, 951-959.

Jensen, H., Steinsland, I., Ringsby, T.H. \& Saether, B.E. (2008). Evolutionary dynamics of a sexual ornament in the house sparrow (Passer domesticus): The role of indirect selection within and between sexes.Evolution , 62, 1275-1293.

Kruuk, L.E.B. (2004). Estimating genetic parameters in natural populations using the "animal model". Philos. Trans. R. Soc. Lond. B. Biol. Sci. , 359, 873-90.

Lande, R. (1982). A Quantitative Genetic Theory of Life History Evolution. Ecology , 63, 607-615.

Lehtonen, J. \& Lanfear, R. (2014). Generation time, life history and the substitution rate of neutral mutations. Biol. Lett. , 10, 3-6.

Liker, A. \& Szekely, T. (2005). Mortality costs of sexual selection and parental care in natural populations of birds. Evolution (N. Y). , 59, 890-897.

MacArthur, R.H. \& Wilson, E.O. (1967). The Theory of Island Biogeography . Landmarks in Biology Series. Princeton University Press.

McGraw, J.B. \& Caswell, H. (1996). Estimation of Individual Fitness from Life-History Data. Am. Nat. , $147,47-64$. 
Metcalfe, N.B. \& Monaghan, P. (2003). Growth versus lifespan: perspectives from evolutionary ecology. Exp. Gerontol. , 38, 935-940.

Niskanen, A.K., Billing, A.M., Holand, H., Hagen, I.J., Araya-Ajoy, Y.G., Husby, A., et al. (2020). Consistent scaling of inbreeding depression in space and time in house sparrows. Proc. Natl. Acad. Sci. , in press.

van Noordwijk, A. \& de Jong, G. (1986). Acquisition and allocation of resources: their influence on variation in life history tactics.Am. Nat. , 128, 137-142.

Parn, H., Ringsby, T.H., Jensen, H., Saether, B.-E., Parn, H., Ringsby, T.H., et al. (2012). Spatial heterogeneity in the effects of climate and density-dependence on dispersal in a house sparrow metapopulation. Proc. R. Soc. B Biol. Sci. , 279, 144-152.

Pianka, E.R. (1970). On r- and K-Selection. Am. Nat. , 104, 592-597.

van de Pol, M. \& Wright, J. (2009). A simple method for distinguishing within- versus between-subject effects using mixed models. Anim. Behav. , 77, 753-758.

R Core Team. (2015). R: A language and environment for statistical computing.

Roff, D.A. (1993). Evolution Of Life Histories: Theory and Analysis . The Evolution of Life Histories: Theory and Analysis. Springer US.

Saether, B.-E. \& Engen, S. (2015). The concept of fitness in fluctuating environments. Trends Ecol. Evol. , $30,273-281$.

Saether, B.-E., Lande, R., Engen, S., Weimerskirch, H., Lillegard, M., Altwegg, R., et al. (2005). Generation time and temporal scaling of bird population dynamics. Nature , 436, 99-102.

Stearns, S.C. (1976). Life-History Tactics: A Review of the Ideas.Q. Rev. Biol. , 51, 3-47.

Stearns, S.C. (1992). The Evolution of Life Histories . OUP Oxford.

Steiner, U.K., Tuljapurkar, S. \& Coulson, T. (2014). Generation time, net reproductive rate, and growth in stage-age-structured populations.Am. Nat. , 183, 771-783.

Stubberud, M.W., Myhre, A.M., Holand, H., Kvalnes, T., Ringsby, T.H., Saether, B.E., et al. (2017). Sensitivity analysis of effective population size to demographic parameters in house sparrow populations. Mol. Ecol. , 26, 2449-2465.

Wright, J., Bolstad, G.H., Araya-Ajoy, Y.G. \& Dingemanse, N.J. (2018). Life-history evolution under fluctuating density-dependent selection and the adaptive alignment of pace-of-life syndromes. Biol. Rev. , 7.

Wright, J., Bolstad, G.H., Araya-Ajoy, Y.G. \& Dingemanse, N.J. (2019). Life-history evolution under fluctuating density-dependent selection and the adaptive alignment of pace-of-life syndromes. Biol. Rev. , 94, 230-247.

Table 1. Univariate mixed-effect model results on the sources of variation in life history traits measuring the timing of reproduction of 1052 individual house sparrows. Only individuals that produced at least one recruit were included in the analyses. We present point estimates and credible intervals in parenthesis.

\begin{tabular}{|c|c|c|c|c|c|c|}
\hline Parameter & $\begin{array}{l}\text { Generation } \\
\text { time (years) }\end{array}$ & $\begin{array}{l}\text { First age } \\
\text { (years) }\end{array}$ & $\begin{array}{l}\text { Lifespan } \\
\text { (years) }\end{array}$ & $\begin{array}{l}\text { Reproduction } \\
\text { rate } \\
\text { (recruits/year) }\end{array}$ & $\begin{array}{l}\text { Life. rep. } \\
\text { success } \\
\text { (recruits) }\end{array}$ & $\begin{array}{l}\text { Growth rate } \\
\text { (recruits) }\end{array}$ \\
\hline
\end{tabular}

Fixed

effects

Intercept

\begin{tabular}{|c|c|c|}
\hline $\begin{array}{l}6(1.22, \\
5)\end{array}$ & $\begin{array}{l}1.08(0.69 \\
1.55)\end{array}$ & $\begin{array}{l}2.65(1.66 \\
3.54)\end{array}$ \\
\hline
\end{tabular}




\begin{tabular}{lllllll} 
Sex (males) & $0.20(0.10$, & $0.21(0.12$, & $0.22(0.07$, & $-0.10(-0.21$, & $0.14(-0.09$, & $-0.05(-0.15$, \\
& $0.30)$ & $0.30)$ & $0.38)$ & $0.00)$ & $0.38)$ & $0.05)$ \\
$\begin{array}{l}\text { Random } \\
\text { effects }\end{array}$ & & & & & \\
Population & $0.02(0.00$, & $0.03(0.00$, & $0.15(0.01$, & $0.00(0.00$, & $0.11(0.00$, & $0.01(0.00$, \\
& $0.06)$ & $0.08)$ & $0.43)$ & $0.01)$ & $0.32)$ & $0.04)$ \\
Year & $0.09(0.01$, & $0.03(0.00$, & $0.36(0.09$, & $0.21(0.08$, & $0.14(0.00$, & $0.00(0.00$, \\
& $0.13)$ & $0.06)$ & $0.74)$ & $0.38)$ & $0.35)$ & $0.01)$ \\
Population: & $0.02(0.00$, & $0.01(0.00$, & $0.04(0.00$, & $0.00(0.00$, & $0.05(0.00$, & $0.03(0.00$, \\
year & $0.05)$ & $0.03)$ & $0.12)$ & $0.01)$ & $0.18)$ & $0.06)$ \\
Residual & $0.71(0.67$, & $0.60(0.55$, & $1.71(1.65$, & $0.26(0.24$, & $4.45(4.20$, & $0.71(0.65$, \\
& $0.77)$ & $0.65)$ & $1.97)$ & $0.28)$ & $5.05)$ & $0.78)$ \\
\hline
\end{tabular}

Table 2. Covariation between life-history traits of 1052 house sparrows estimated in the lower diagonal and correlations in the upper diagonal. We present point estimates and credible intervals in parenthesis.

\begin{tabular}{|c|c|c|c|c|c|c|}
\hline & $\begin{array}{l}\text { Generation } \\
\text { time }\end{array}$ & First age & Lifespan & $\begin{array}{l}\text { Reproduction } \\
\text { rate }\end{array}$ & $\begin{array}{l}\text { Life. rep. } \\
\text { success }\end{array}$ & Growth rate \\
\hline \multirow{2}{*}{$\begin{array}{l}\text { Generation } \\
\text { time }\end{array}$} & - & 0.76 & 0.82 & -0.20 & 0.44 & -0.03 \\
\hline & & $\begin{array}{l}(0.73, \\
0.78)\end{array}$ & $\begin{array}{l}(0.80 \\
0.84)\end{array}$ & $\begin{array}{l}(-0.26 \\
-0.14)\end{array}$ & $\begin{array}{l}(0.39 \\
0.49)\end{array}$ & $\begin{array}{l}(-0.09 \\
0.02)\end{array}$ \\
\hline First age & $\begin{array}{l}0.50(0.45 \\
0.57)\end{array}$ & - & $\begin{array}{l}0.46(0.41, \\
0.52)\end{array}$ & $\begin{array}{l}-0.33(-0.39, \\
-0.27)\end{array}$ & $\begin{array}{l}-0.02(-0.07 \\
0.04)\end{array}$ & $\begin{array}{l}-0.26(-0.31 \\
-0.20)\end{array}$ \\
\hline Lifespan & $\begin{array}{l}1.11(1.03, \\
1.24)\end{array}$ & $\begin{array}{l}0.51(0.49, \\
0.59)\end{array}$ & - & $\begin{array}{l}-0.27(-0.32, \\
-0.21)\end{array}$ & $\begin{array}{l}0.51(0.47 \\
0.57)\end{array}$ & $\begin{array}{l}0.08(0.02, \\
0.14)\end{array}$ \\
\hline $\begin{array}{l}\text { Reproduction } \\
\text { rate }\end{array}$ & $\begin{array}{l}-0.15(-0.21 \\
-0.10)\end{array}$ & $\begin{array}{l}-0.20(-0.16 \\
-0.24)\end{array}$ & $\begin{array}{l}-0.32(-0.41, \\
-0.26)\end{array}$ & - & $\begin{array}{l}0.54(0.49, \\
0.58)\end{array}$ & $\begin{array}{l}0.82(0.79, \\
0.89)\end{array}$ \\
\hline $\begin{array}{l}\text { Life. rep. } \\
\text { success }\end{array}$ & $\begin{array}{l}0.89(0.76, \\
1.03)\end{array}$ & $\begin{array}{l}-0.06(-0.11 \\
0.07)\end{array}$ & $\begin{array}{l}1.69(1.47, \\
1.91)\end{array}$ & $\begin{array}{l}0.97(0.84, \\
1.10)\end{array}$ & - & $\begin{array}{l}0.68(0.64, \\
0.71)\end{array}$ \\
\hline Growth rate & $\begin{array}{l}-0.02(-0.09 \\
0.02)\end{array}$ & $\begin{array}{l}-0.16(-0.20 \\
-0.12)\end{array}$ & $\begin{array}{l}0.11(0.03, \\
0.19)\end{array}$ & $\begin{array}{l}0.58(0.52, \\
0.64)\end{array}$ & & \\
\hline
\end{tabular}

Table 3. Results for the age- and density-dependent mixed-effect models on reproduction (number of recruits produced per year, Poisson) and survival (binomial), based upon 5247 individual breeding attempts for 2729 individual house sparrows. Age is modelled as a two-level categorical variable; first year breeding versus older $\left(>1^{\text {st }}\right.$ year $)$.

\begin{tabular}{lll}
\hline Parameter & Reproduction & Survival \\
A) Fixed effects & & \\
Intercept & $-1.03(-1.24,-0.82)$ & $0.02(-0.27,0.28)$ \\
Age $\left(>1^{\text {st }}\right.$ year $)$ & $0.32(0.21,0.43)$ & $0.13(-0.05,0.32)$ \\
Sex (male) & $-0.21(-0.34,-0.08)$ & $0.32(0.15,0.50)$ \\
Age: sex & $0.25(0.08,0.40)$ & $-0.13(-0.40,0.14)$ \\
Mean pop size & $0.10(-0.04,0.24)$ & $-0.05(-0.25,0.19)$ \\
Relative pop size & $-0.14(-0.20,-0.01)$ & $-0.02(-0.22,0.15)$ \\
B) Random effects & & \\
Individual & $0.39(0.29,0.49)$ & $0.00(0.00,0.03)$ \\
Population & $0.02(0.00,0.07)$ & $0.05(0.00,0.20)$ \\
Year & $0.04(0.00,0.11)$ & $0.07(0.04,0.18)$
\end{tabular}




\begin{tabular}{lll} 
Population: year & $0.20(0.11,0.30)$ & $0.25(0.13,0.40)$ \\
Residual & $0.24(0.17,0.35)$ & $1(1,1)$ \\
\hline
\end{tabular}

Table 4. Mixed-effect model results explaining variation in the age of successfully reproducing parents. Model 1A focuses on the effects of mean fitness, and model 2A focuses on the effect of population size. B Models denote results after correcting for the age structure of the population. In bold we present the estimates where the confidence intervals did not overlap zero.

\begin{tabular}{|c|c|c|c|c|}
\hline $\begin{array}{l}\text { Parameter } \\
\text { Fixed effects }\end{array}$ & Model 1A & Model 1B & Model 2A & Model 2B \\
\hline Intercept & $2.88(2.42,2.38)$ & $0.42(-0.21,0.89)$ & $2.22(1.88,2.32)$ & $0.07(-0.25,0.36)$ \\
\hline Sex (females) & $-0.43(-0.96,0.08)$ & $-0.23(-0.64,0.16)$ & $0.12(-0.32,0.07)$ & $0.02(-0.11,0.19)$ \\
\hline$w$ & $-0.75(-1.18,-0.25)$ & $-0.34(-0.62,-0.002)$ & & \\
\hline$w: \operatorname{sex}$ & $0.35(-0.16,0.97)$ & $0.27(-0.15,0.62)$ & & \\
\hline Mean adult age & & $1.05(0.85,1.20)$ & & $1.06(0.96,1.21)$ \\
\hline Mean pop size & & & $-0.06(-0.24,0.14)$ & $0.00(-0.08,-0.06)$ \\
\hline Relative pop size & & & $0.13(-0.07,0.32)$ & $0.07(-0.07,0.19)$ \\
\hline $\begin{array}{l}\text { Relative pop size: sex } \\
\text { Random effects }\end{array}$ & & & $-0.08(-0.32,0.22)$ & $-0.06(-0.25,0.11)$ \\
\hline Population & $0.07(0.00,0.20)$ & $0.01(0.0,0.06)$ & $0.00(0.00,0.02)$ & $0.02(0.0,0.06)$ \\
\hline Year & $0.02(0.00,0.05)$ & $0.00(0.00,0.01)$ & $0.00(0.00,0.02)$ & $1.17(0.47,2.03)$ \\
\hline Residual & $0.43(0.34,0.52)$ & $0.20(0.16,0.24)$ & $0.24(0.19,0.29)$ & $0.71(0.67,0.77)$ \\
\hline
\end{tabular}



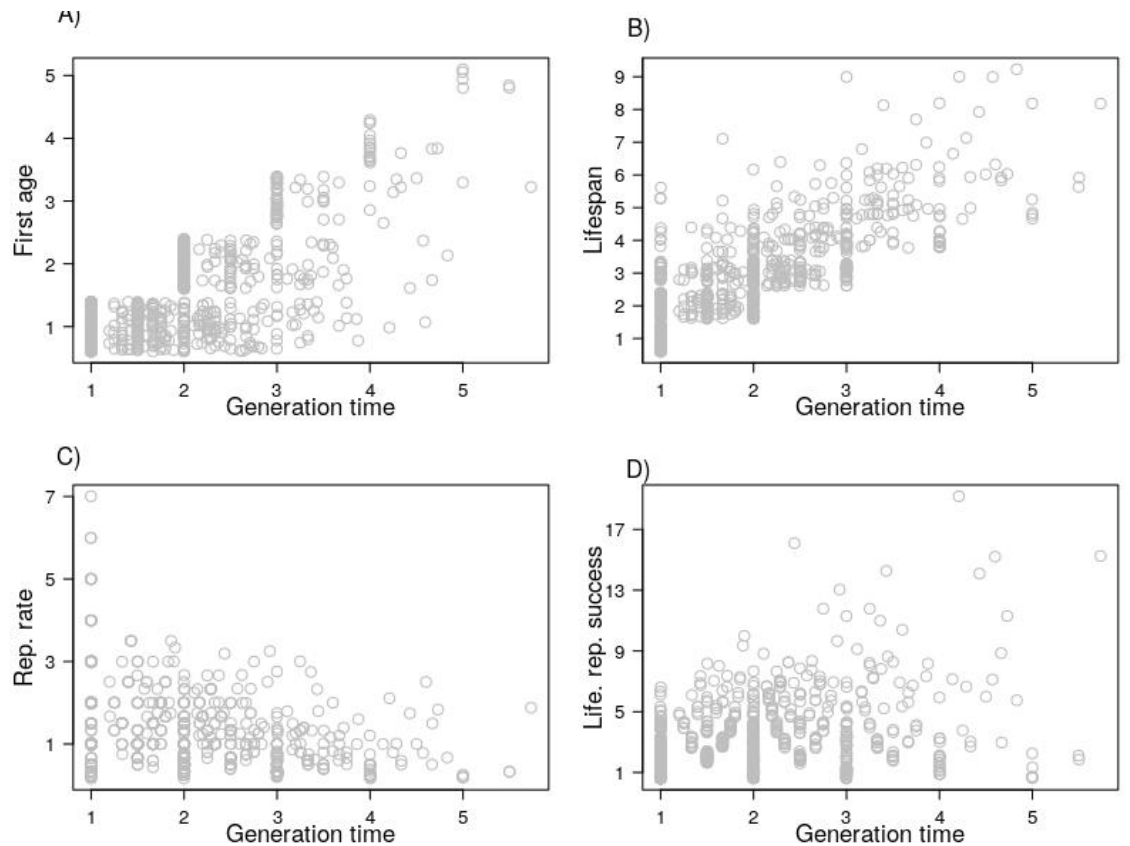

E)

F)
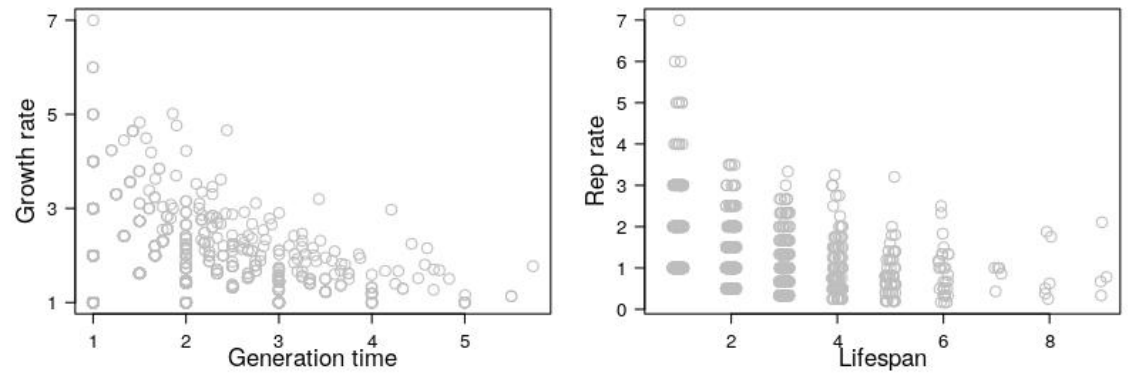

Figure 1. The relationships between generation time and first age at reproduction (A), lifespan (B), reproduction rate $(\mathrm{C})$, lifetime reproductive success $(\mathrm{D})$, and individual expected growth rate (E). In addition, the relationship between lifespan and reproduction rate $(F)$. Reproductive rate is the number of recruits produced per year by all individuals that managed to produce at least one recruiting offspring.
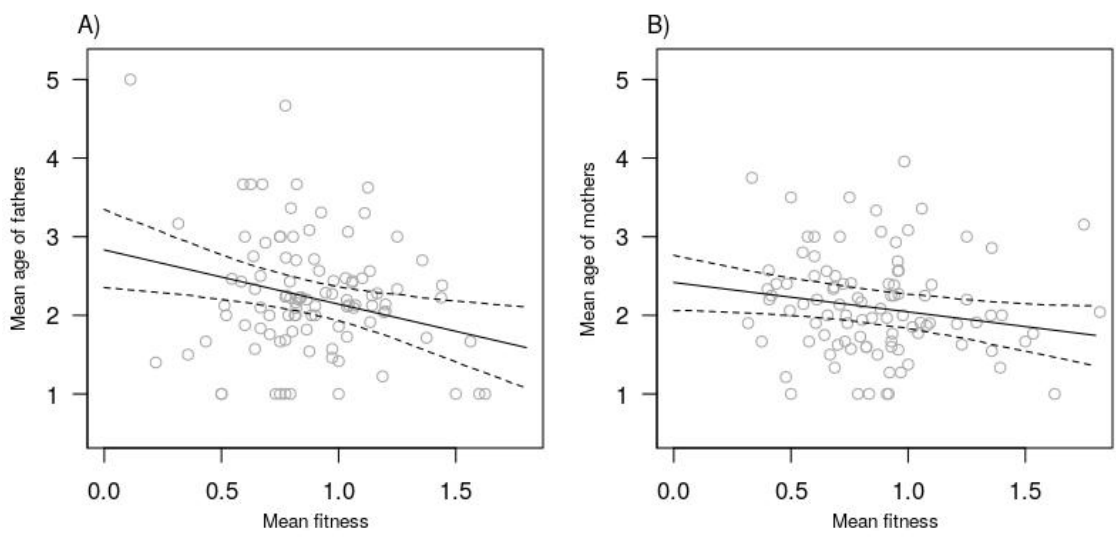
Figure 2. The relationship between mean fitness in the population and mean age of successful reproduction for (A) males and (B) females. Each dot represents each population in each year. Lines are the predicted mean values and dotted lines the $95 \%$ credible intervals.

\section{Appendix S1. Sample sizes, formulas and statistical models' equations.}

\section{A) Sample sizes}

Table S1. Sample sizes and time periods for each of the studied populations. For the time period of individual life histories, the first year refers to the earliest cohort year included in the analyses and we included only individuals that were known to be dead after the last year (i.e. where not recorded in the next two breeding seasons). For age and density dependent analyses, the time period refers to all individuals present during those years.

\begin{tabular}{lllll}
\hline & $\begin{array}{l}\text { Individual } \\
\text { life-histories }\end{array}$ & $\begin{array}{l}\text { Individual } \\
\text { life-histories }\end{array}$ & $\begin{array}{l}\text { Age- and density- } \\
\text { dependence } \\
\text { models }\end{array}$ & $\begin{array}{l}\text { Age- and density- } \\
\text { dependence } \\
\text { models }\end{array}$ \\
\hline $\begin{array}{l}\text { Population } \\
\text { Inner-farm }\end{array}$ & Time period & Individuals & Time period & Individuals \\
$\begin{array}{l}\text { Nesøy } \\
\text { Gjerøy }\end{array}$ & $1997-2012$ & 40 & $1998-2012$ & 122 \\
Hestmannøy & $1997-2012$ & 184 & $1998-2012$ & 529 \\
Indre Kvarøy & $1997-2012$ & 432 & $1998-2012$ & 1014 \\
Aldra & $1997-2012$ & 134 & $1998-2012$ & 344 \\
Outer-non-farm & $1997-2012$ & 83 & $1998-2012$ & 181 \\
Myken & & & & \\
Selvær & $2003-2011$ & 16 & $2004-2011$ & 68 \\
Træna & $2002-2012$ & 77 & $2003-2012$ & 223 \\
Total & $2002-2012$ & 86 & $2003-2012$ & 248 \\
\hline
\end{tabular}

\section{B) Estimates of individual life-history traits}

Based on the pedigree, we used the number of recruits produced by each individual in each year to calculate all the elements of the individual projection matrices and derive some key life-history traits, such as individual expected growth rate and generation time (McGraw \& Caswell 1996). To calculate an individual's generation time, it must have produced a recruit. Thus, only individuals that produced at least one recruit during their lifetime were considered in these analyses of individual life-histories, resulting in a total of 1052 individuals (552 females and 500 males, see Table S1 for more details).

To estimate the projection matrix $\mathrm{A}^{(i)}$ of individual $i$ we need to know the number of successful recruits it produced $\left(F_{h}^{(i)}\right)$ at each age $h$ and the age when it died $d$ (McGraw \& Caswell 1996). Where survival $S_{h}^{(i)}$ will be equal to one for all $h=(1,2, \ldots, \mathrm{d}-1)$ and zero for $h=d$ :

$A^{(i)}=$

$$
\left[\begin{array}{cccc}
F_{1}^{(i)} & a m p ; F_{2}^{(i)} & a m p ; \cdots & a m p ; F_{d}^{(i)} \\
S_{1}^{(i)} & a m p ; 0 & a m p ; \cdots & a m p ; 0 \\
& a m p ; \cdot & a m p ; & a m p ; \\
0 & a m p ; & a m p ; S_{d-1}^{(i)} & a m p ; 0
\end{array}\right] \text {. (eq. S1a) }
$$

Once an individual transition matrix $A^{(i)}$ is formed, the dominant eigenvalue $\lambda^{(i)}$ of this matrix measures the asymptotic population growth rate for a collection of individuals with the propensities to survive and reproduce equal to individual $i$ (McGraw \& Caswell 1996); that is, it is an estimate of the expected growth 
rate of a population consisting of individuals with the characteristics of individual $i$ (i.e. expected individual growth rate):

$1=\sum_{h=1}^{d^{(i)}} F_{h}^{(i)}\left(\lambda^{(i)}\right)^{-h}$. (eq. S1b)

A similar life-history measure can be defined as an individual's reproductive rate $\left(r^{(i)}\right)$, which is the mean number of recruits an individual produced per year:

$r^{(i)}=\frac{1}{d^{(i)}} \sum_{h=1}^{d^{(i)}} F_{h}^{(i)}$. (eq. S2)

The lifetime reproductive success $R^{(i)}$ of individual $i$ can be estimated as the sum of the fecundities at each age $h$ :

$R^{(i)}=\sum_{h=1}^{d^{(i)}} F_{h}^{(i)}$. (eq. S3)

The first age at reproduction was calculated as the first age hat which individual $i$ managed to successfully produce a recruit $\left(F_{h}^{(i)}>0\right)$, and the lifespan was the age $d$ when individual $i$ was last observed. We estimated an individual measure of generation time $T^{(i)}$ as the weighted mean age of an individual when it reproduced as:

$T^{(i)}=\frac{\sum_{h=1}^{d^{(i)}} \mathrm{hF}_{h}^{(i)}}{\sum_{h=1}^{d^{(i)}} F_{h}^{(i)}}$. (eq. S4)

(C) Modelling individual life-histories

The model describing life-history variation presented in table 1 in a mixed-effect model form is:

$y_{\mathrm{gjki}}=u_{g}+\beta_{1 g} S_{i}+\beta_{2 g} H_{i}+C_{\mathrm{gj}}+P_{\mathrm{gk}}+e_{\mathrm{gjki}}$, (eq. S5)

where $y_{\mathrm{gjk} i}$ represents life-history trait $g$ (generation time, first age at reproduction, reproduction rate, lifespan, lifetime reproductive success or individual growth rate) of individual $i$ in cohort $j$ and population $k$ - Here, $u_{g}$ represents the statistical intercept for the model, which is the estimated mean life-history trait for the reference category. $\beta_{1 g}$ is the coefficient reflecting average sex differences in the life-history trait $g$, where $S_{i}$ represents the sex of individual i ( 0 for females; 1 for males). $\beta_{2 g}$ is the coefficient describing the average life-history differences between the type of island where an individual was breeding ( 0 for inner farm islands; 1 for outer non-farm islands). $C_{\mathrm{gj}}$ represents the cohort effects $(\mathrm{n}=16)$ on the life-history traits, and $P_{\mathrm{gk}}$ represents the population differences $(\mathrm{n}=8)$ in the average life-history trait. In this case, $e_{\mathrm{gjki}}$ reflects individual differences $(\mathrm{n}=1232)$ in the life-history traits, because there are no repeated measures for each individual as the life-history traits are estimated for the whole life. Cohort specific values $\left(C_{\mathrm{gj}}\right)$, population specific values $\left(P_{\mathrm{gk}}\right)$ and individual specific values $e_{\mathrm{gjki}}$, were all assumed to come from separate normal distributions for each life-history trait with variance $V_{C_{g}}, V_{P_{g}}$ and $V_{e_{g}}$.

(C) Age- and density-dependent reproduction and survival

For the age- and density-dependent models, we utilized annual data on reproduction and survival for all individuals present within the studied time periods (Table S1). In total, there were 5247 records from 2729 individuals (1325 females and 1361 males). We studied how annual measures of reproduction and survival resulted in the observed means and (co)variance of individual life-histories by building age- and densitydependent reproduction and survival models. First, we estimated the age- and sex-specific annual number of recruits using univariate mixed-effect models on the following form:

$\eta_{\mathrm{ghijk}}=c_{g}+\beta_{1 g} S_{i}+\beta_{2 g} a_{\mathrm{hi}}+\beta_{3 g} a_{\mathrm{hi}} S_{i}+\beta_{4 g} n_{k}+\beta_{5 g}\left(n_{\mathrm{jk}}-n_{k}\right)+I_{\mathrm{gi}}+Y_{\mathrm{gj}}+P_{\mathrm{gk}}+e_{\text {ghijk }}$, (eq. S6)

We model survival and annual number of recruits at age $h$ of individual $i$ breeding in year $j$ on population $k$, where $\eta_{1 \mathrm{hijk}}=\operatorname{logit}\left(\right.$ survival) and $\eta_{2 \mathrm{hijk}}=\log ($ number of recruits). Both variables were modelled with the same fixed and random effect structure, however the residual error for survival was assumed to be that of a binomial distribution and thus its variance was fixed to 1 , whereas the residual variance for the number 
of recruits was assumed to be that of an over-dispersed Poisson distribution, where we estimated the overdispersion component. $\beta_{1 g}$ represents the average sex differences in yearly survival and reproduction, and $\beta_{2 g}$ represents age-specific survival and reproduction. Where $g$ denotes whether the effects are for reproduction or survival. We treated age as a two-level categorical variable (first year breeders versus older individuals) and also fitted an interaction with sex, as we were expecting sex-specific $\left(\beta_{3 g}\right)$ patterns of age-dependent reproduction and survival (Stubberud et al. 2017). These models had also as fixed effect $\left(\beta_{4 g}\right)$ for the mean population size $\left(n_{k}\right)$ of population $(k)$ and the effect $\left(\beta_{5 g}\right)$ of yearly deviations from the mean population size in number of individuals $\left(n_{\mathrm{jk}}-n_{k}\right)$. This within-subject centering approach allowed us to model density regulation accounting for differences in the mean population size between populations, and allowed us to test for any spatial versus temporal effects of population size in recruitment and survival (van de Pol \& Wright 2009). Here, year-specific values $\left(Y_{\mathrm{gj}}\right)$, population-specific values $\left(P_{\mathrm{gk}}\right)$, individual-specific values $I_{\mathrm{gi}}$, and within-individual residual deviations $e_{\text {ghijk }}$, were all assumed to come from separate normal distributions for each life-history trait with variances $V_{Y_{g}}, V_{P_{g}}, V_{I_{g}}$, and $V_{e_{g}}$.

Second, we fitted a multivariate mixed-effects model, where we estimated the covariance between yearly survival and recruit production at the individual and residual levels:

$$
\begin{aligned}
& {\left[\begin{array}{l}
I_{s} \\
I_{f}
\end{array}\right] \sim \operatorname{mvn}\left(0, \mathbf{V}_{\mathbf{I}}\right) ; \mathbf{V}_{\mathbf{I}}=} \\
& {\left[\begin{array}{cc}
\mathbf{V}_{\mathbf{I}_{1}} & a m p ; \\
\mathbf{C}_{\mathbf{I}_{12}} & a m p ; \mathbf{V}_{\mathbf{I}_{2}}
\end{array}\right], \text { (eq. S3a) }} \\
& {\left[\begin{array}{c}
e_{s} \\
e_{f}
\end{array}\right] \sim \operatorname{mvn}\left(0, \mathbf{V}_{\mathbf{e}}\right) ; \mathbf{V}_{\mathbf{e}}=\left[\begin{array}{cc}
\mathbf{V}_{\mathbf{e}_{1}} & a m p ; \\
\mathbf{C}_{\mathbf{e}_{12}} & a m p ; \mathbf{V}_{\mathbf{e}_{2}}
\end{array}\right], \text { (eq. S3b) }}
\end{aligned}
$$

where $I_{s}$ and $I_{f}$ represent an individuals average survival and annual number of recruit production in the latent scales, while $e_{s}$ and $e_{f}$ represent the deviations of each breeding season for each individuals mean values, also in the latent scale. $V_{I}$ represents the among individual variance covariance matrix, with elements $V_{I_{1}}$, $V_{I_{2}}$ and $C_{I_{12}}$, representing among-individual variance in survival, annual reproduction and their covariance, respectively. Whereas $\mathbf{V}_{\mathbf{e}}$ represents the within-individual variance covariance matrix, with elements $V_{e_{1}}$, $V_{e_{2}}$ and $C_{e_{12}}$, representing within-individual variance in survival, annual reproduction and their covariance, respectively. Note that $V_{e_{1}}$ was fixed to one by convention.

\section{(D) Effects of population dynamics on the mean age of reproduction}

To explore how population dynamics affected the mean age of parents of recruits each year in each population $\left(T_{j k}\right)$, we utilized annual data on reproduction and survival for all adult individuals $i$ present within the studied time periods (Table S1). From this data we estimated the weighted mean age of the parents reproducing in year $j$ and population $k$ as:

$T_{\mathrm{jk}}=\frac{\sum_{i=1}^{n_{\mathrm{jk}}} \mathrm{hF}^{(i j k)}}{\sum_{i=1}^{n_{j k}} F^{(i j k)}}$, (eq. S4)

where $h$ is the age and $F$ is the number of recruiting offspring produced by individual $i$ in population $j$ in yeark. The sum is taken for all individuals breeding in year $j$ in population $k\left(n_{\mathrm{jk}}\right)$. We estimated the mean age at reproduction in a population each year for males and females separately. We then fitted a mixed-effect model that had as response variable the mean age of reproducing individuals in a given year in a given population $\left(T_{j k}\right)$, and as fixed effects sex and the mean and annual deviations of population size to distinguish between effects of spatial versus temporal fluctuations in population size on the mean age at reproduction of a population.

To further examine how $T_{j k}$ was related to the ecological factors determining population growth, we fitted another mixed-effect model where the mean age at reproduction $\left(T_{j k}\right)$ was fitted as a response variable and the mean fitness of the population in each year and sex as fixed effects. We estimated the fitness of 
individual $i$ in year $j$ as survival plus half the number of recruits to the next year, because, in the absence of dispersal, this metric of fitness accounts for sexual reproduction and directly connects to local population dynamics (Sæther \& Engen 2015):

$w_{\mathrm{ij}}=S_{\mathrm{ij}}+\frac{1}{2} F_{\mathrm{ij}} \cdot($ eq. S 5 )

The average fitness of a population each year was thus estimated as the mean fitness of all individuals breeding in a year in a population:

$w_{\mathrm{jk}}=\frac{1}{N_{\mathrm{jk}}} \sum_{i=1}^{n_{\mathrm{jk}}} w_{\mathrm{hij}}$, (eq. S6)

where the sum is taken for all individuals breeding in year $j$ in population $k$. Here, $n$ is the number of adults breeding in year $j$ in population $k$. Importantly, $w_{\mathrm{jk}}$ will determine the changes in population size across years that are not caused by immigration and emigration, but it could be affected by recapture probabilities. The mean fitness in the population directly connects to the expected population growth and should reflect current levels of competition in the population (Sæther \& Engen 2015), either because of variation in environmental conditions and/or due to variation in population density relative to the amount of resources. To control for the effects of age structure in determining the mean age at reproduction, we also fitted the two above mentioned models including the mean age of all the adults breeding in the population as an additional fixed effect.

We modelled the mean age of the successfully reproducing parents $T_{\mathrm{jk}}$ in year $j$ in population $k$ as

$T_{\mathrm{jk}}=c+\beta_{1} S_{i}+\beta_{2} n_{k}+\beta_{3}\left(n_{\mathrm{jk}}-n_{k}\right)+Y_{j}+P_{k}+e_{\mathrm{jk}}($ eq. S 7$)$

where $c$ is the average age of the successfully reproducing parents in the meta-population. $\beta_{1}$ is the coefficient reflecting average sex differences in the mean age at reproduction $\left(S_{i}=0\right.$ for females; $S_{i}=1$ for males $)$. This model had also as fixed effect $\left(\beta_{2}\right)$ for the mean population size $\left(n_{k}\right)$ of population $(k)$ and the effect $\left(\beta_{3}\right)$ of yearly deviations from the mean population size in number of individuals $\left(n_{\mathrm{jk}}-n_{k}\right)$. This within-subject centering approach allowed us to model density regulation accounting for differences in the mean population size between populations, and allowed us to test for any spatial versus temporal effects of population size in the mean age of the successfully reproducing parents (van de Pol \& Wright 2009). Here, year-specific values $\left(Y_{j}\right)$, population-specific values $\left(P_{k}\right)$, and within-population residual deviations $e_{\mathrm{jk}}$, were all assumed to come from separate normal distributions for each life-history trait with variances $V_{Y}, V_{P}$ and $V_{e_{g}}$. We also fitted a model with the same random effect structure, but the fixed effect structure differed in that instead of the effect of the average population size $\left(\beta_{2}\right)$ and the yearly deviations from the average population size $\left(\beta_{3}\right)$, we fitted the effect $\left(\beta_{4}\right)$ of the mean fitness $w_{\mathrm{jk}}$ of population $k$ in year $j$ as another fixed effect. To further corroborate the results we included the mean age of all in the individuals we inferred to be present in each population in each year to account for potential effects of age structure $\left(\beta_{5}\right)$ in the results in both of the models mentioned in this section (D).

\section{Appendix S2. Complementary analyses and figures.}

Table S2 . Animal model full results. Presented the estimates of the MCMCglmm and ASREML models. We present the point estimates, and the standard error or $95 \%$ credible intervals (CI) in parenthesis.

\begin{tabular}{lll}
\hline Parameter & AsReml & MCMCglmm \\
Fixed effects & $\beta(S E)$ & $\beta(95 \% C I)$ \\
Intercept & $1.48(0.11)$ & $1.48(1.23,1.70)$ \\
Sex (males) & $0.15(0.05)$ & $0.16(0.06,0.26)$ \\
Random effects & $\sigma^{2}(S E)$ & $\sigma^{2}(95 \% C I)$ \\
Cohort & $0.08(0.03)$ & $0.10(0.03,0.23)$ \\
Additive genetic & $0.04(0.03)$ & $0.04(0.00,0.08)$ \\
Residual & $0.71(0.04)$ & $0.71(0.62,0.78)$ \\
\hline
\end{tabular}


Table S3 . Principal components analyses applied to the life-history trait covariance matrix (Table 2 main text).

\begin{tabular}{lll}
\hline Variable & PC1 & PC2 \\
\hline Variance exp. & 0.73 & 0.26 \\
Loadings & & \\
First age & -0.08 & 0.29 \\
Generation time & 0.30 & 0.69 \\
Lifespan & 0.12 & 0.40 \\
Reproductive rate & 0.16 & -0.42 \\
LRS & 0.89 & -0.11 \\
Growth rate & 0.23 & -0.29 \\
\hline
\end{tabular}
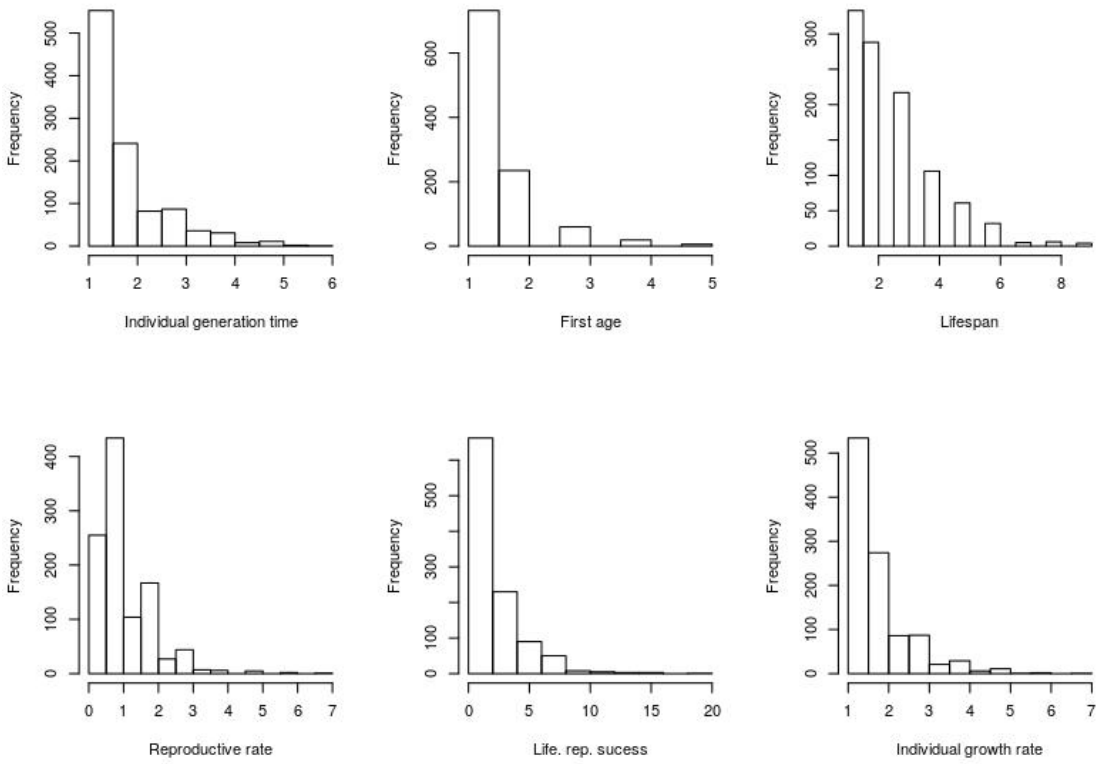

Figure S1. The distribution of individual life-history metrics in the house sparrow metapopulation. 

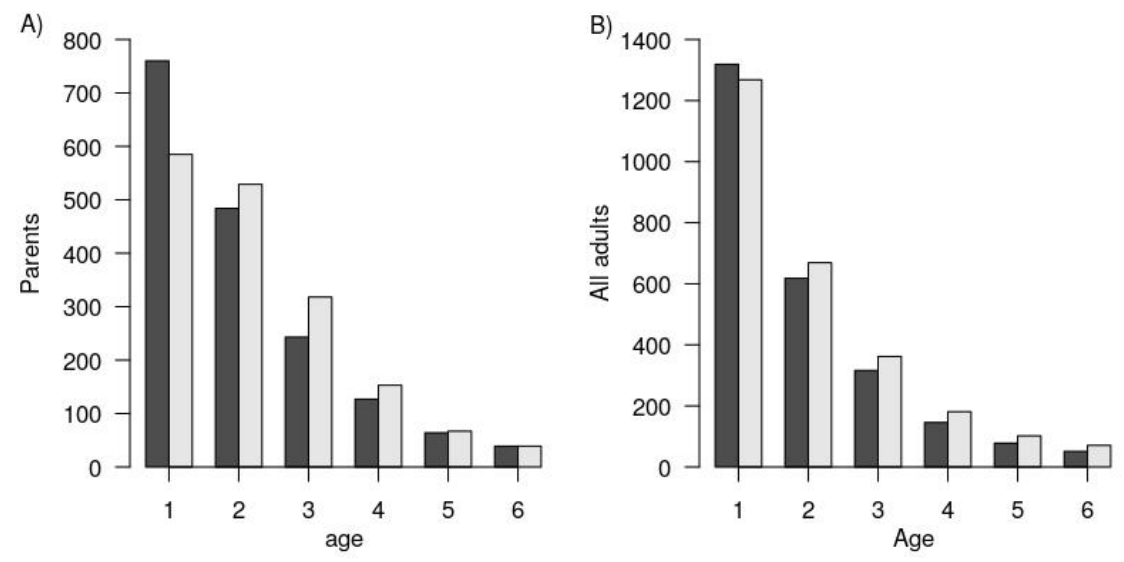

Figure S2 . A) Age distribution for the parents for each recruit split between mothers (white) and fathers (white). B) Age distribution of all the adult males (black) and females (white) in the population.

Appendix S3. A simulation study based upon the demographic parameters estimated for the studied house sparrow metapopulation

\section{(A) Statistical models and simulation equations}

We used simulations to study how the observed pattern of covariation among life-histories, was caused by the among- and within individual variation in the annual reproduction and survival. More specifically we wanted to assess how the negative covariance between life span, and reproductive rate and generation time and reproductive rate could be caused by a positive covariance between annual reproduction and survival. To this end, we used the estimated parameters in the models of annual survival and reproduction (Table 3 ) to simulate individual life-histories, and from that, estimate the patterns of covariation between individual life-histories that arise from the annual estimates of annual survival and recruit production.

We used a modified version of equation 2 to simulate individual life-histories through time.

$\eta_{\text {ghij }}=c_{g}+\beta_{2 g} a_{\mathrm{hi}}+\beta_{4 g} n_{k}+\beta_{5 g} n_{\mathrm{jk}}+I_{\mathrm{gi}}+Y_{\mathrm{gj}}+e_{\mathrm{ghij}}($ eq. S8)

We simulated annual survival and annual number of recruits at age $h$ of individual $i$ breeding in year $j$. Where $\eta_{1 \text { hijk }}=\operatorname{logit}\left(\right.$ survival) and $\eta_{2 \text { hijk }}=\log$ (number of recruits). The number of recruits and whether an individual survived to the next year was simulated using values for the different components of this equation that were estimated for our population. Where $\beta_{1 g}$ represents the average sex differences in yearly survival and reproduction, and $\beta_{2 g}$ represents age-specific survival and reproduction. Where $g$ denotes whether the effects are for reproduction or survival. As in our analyses, the effect of age was treated as a two-level categorical variable, where first year breeders had lower reproduction compared to older individuals as a function of the estimated parameters in table 2. For simplicity we modelled the male and female population separately based on the sex specific values of survival and reproduction. We ignored population differences and simulated a single meta-population, were population wide differences where simulated as individual differences. Here, year-specific values $\left(Y_{\mathrm{gj}}\right)$ for survival and reproduction were simulated from a normal distribution with variance equal to the one we estimated in our models.

Importantly individual specific values and within individual realization in different years, where simulated from multivariate distributions with variance-covariance matrix $V_{I}$ and $\mathbf{V}_{\mathbf{e}}$, for the among and within individual effects.

$$
\left[\begin{array}{c}
\mathbf{I}_{\mathbf{S}} \\
\mathbf{I}_{\mathbf{f}}
\end{array}\right] \sim \operatorname{mvn}\left(0, \mathbf{V}_{\mathbf{I}}\right) ; \mathbf{V}_{\mathbf{I}}=
$$




$$
\begin{aligned}
& {\left[\begin{array}{cc}
V_{I_{1}} & a m p ; \\
C_{I_{12}} & a m p ; V_{I_{2}}
\end{array}\right],(\text { eq. S9a) }} \\
& {\left[\begin{array}{l}
\mathbf{e}_{\mathbf{s}} \\
\mathbf{e}_{\mathbf{f}}
\end{array}\right] \sim \operatorname{mvn}\left(0, \mathbf{V}_{\mathbf{e}}\right) ; \mathbf{V}_{\mathbf{e}}=\left[\begin{array}{cc}
V_{e_{1}} & a m p ; \\
C_{e_{12}} & a m p ; V_{e_{2}}
\end{array}\right],(\text { eq. S } 9 \mathrm{~b})}
\end{aligned}
$$

where $I_{s}$ and $I_{f}$ represent an individuals average survival and annual number of recruit production in the latent scales, while $e_{s}$ and $e_{f}$ represent the deviations of each breeding season for each individuals mean values, also in the latent scale. Where $V_{I}$ represents the among individual variance covariance matrix, with elements $V_{I_{1}}, V_{I_{2}}$ and $C_{I_{12}}$, representing among-individual variance in survival, annual reproduction and their covariance, respectively. Whereas $\mathbf{V}_{\mathbf{e}}$ represents the within-individual variance-covariance matrix, with elements $V_{e_{1}}, V_{e_{2}}$ and $C_{e_{12}}$, representing within-individual variance in survival, annual reproduction and their covariance, respectively. Note that $V_{e_{1}}$ was fixed to one by convention. Based on the patterns of survival and reproduction we simulated the next year breeding individuals and so on for 20 years. We simulated 1000 metapopulations and estimated the covariance in life-history trade-offs between scenarios of the observed positive covariance, a scenario of negative covariance (opposite sign of what was observed) and no covariance between survival and reproduction

\section{(B) Results}

We found a negative covariance between lifespan and reproductive rate even when there was a positive relationship between lifespan and annual number of recruits (Figure S3A). This covariance was stronger when there was no variation in resources causing a covariance between reproduction and survival (Figure S3B), and even stronger if there would indeed have been a negative covariance between survival and reproduction (Figure S3C).
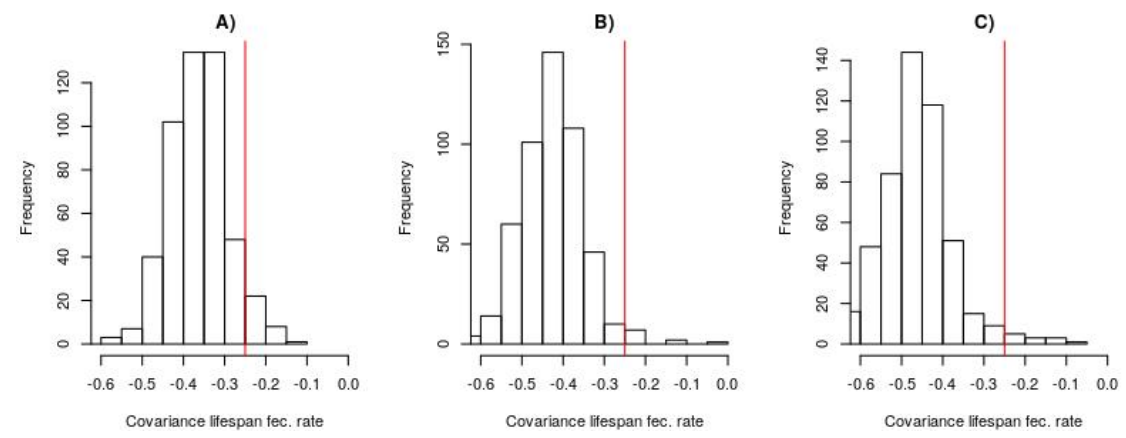

Figure S3. Estimated covariances between lifespan and reproductive rate from 1000 simulations with A) the positive covariance between annual reproduction and survival found in the study (see Results), B) with no covariance, and $\mathrm{C}$ ) with a negative covariance with the same magnitude as the one found in our study of a house sparrow metapopulation. The red line shows the observed mean covariance between lifespan and reproductive rate in our population. 